\title{
Nonperturbative calculation of the probability distribution of plane-wave transmission through a disordered waveguide
}

\author{
S. A. van Langen, P. W. Brouwer, and C. W. J. Beenakker \\ Instituut-Lorentz, University of Leiden, P.O. Box 9506, 2300 RA Leiden, The Netherlands
}

(Received 7 September 1995)

\begin{abstract}
A nonperturbative random-matrix theory is applied to the transmission of a monochromatic scalar wave through a disordered waveguide. The probability distributions of the transmittances $T_{m n}$ and $T_{n}=\Sigma_{m} T_{m n}$ of an incident mode $n$ are calculated in the thick-waveguide limit, for broken time-reversal symmetry. A crossover occurs from Rayleigh or Gaussian statistics in the diffusive regime to lognormal statistics in the localized regime. A qualitatively different crossover occurs if the disordered region is replaced by a chaotic cavity.
\end{abstract}

PACS number(s): 42.25.Bs, 05.45.+b, 72.15.Rn, 78.20.Dj

The statistical properties of transmission through a disordered waveguide have been extensively studied since 1959, when Gertsenshtein and Vasil'ev [1] computed the probability distribution $P(T)$ of the transmittance $T$ of a single-mode waveguide. It turned out to be remarkably difficult to extend this result to the $N$-mode case. Instead of a single transmission amplitude $t$ and transmittance $T=|t|^{2}$, one then has an $N \times N$ transmission matrix $t_{m n}$ and three types of transmittances

$$
T_{m n}=\left|t_{m n}\right|^{2}, \quad T_{n}=\sum_{m=1}^{N}\left|t_{m n}\right|^{2}, \quad T=\sum_{n, m=1}^{N}\left|t_{m n}\right|^{2}
$$

All three transmittances have different probability distributions, which can be measured in different types of experiments: If the waveguide is illuminated through a diffusor, the ratio of transmitted and incident power equals $T / N$, because the incident power is equally distributed among all $N$ modes. (For electrons, $T$ is the conductance in units of $2 e^{2} / h$.) If the incident power is entirely in mode $n$, then the ratio of transmitted and incident power equals $T_{n}$. For $N \gg 1$ this corresponds to illumination by a plane wave. Finally, $T_{m n}$ measures the speckle pattern (the fraction of the power incident in mode $n$ which is transmitted into mode $m$ ).

The complexity of the multimode case is due to the strong coupling of the modes by multiple scattering. While in the single-mode case the localization length $\xi$ is of the same order of magnitude as the mean free path $l$, the mode coupling increases $\xi$ by a factor of $N$. If $N \gg 1$, a waveguide of length $L$ can be in two distinct regimes: the diffusive regime $l \ll L \ll N l$ and the localized regime $L \gg N l$. The average of each of the three transmittances decays linearly with $L$ in the diffusive regime and exponentially in the localized regime. In an important development, Nieuwenhuizen and van Rossum [2] (and more recently Kogan and Kaveh [3]) succeeded in computing the probability distributions $P\left(T_{m n}\right)$ and $P\left(T_{n}\right)$ for plane-wave illumination in the diffusive regime. The former is exponential (Rayleigh's law) with nonexponential tails, while the latter is Gaussian with non-Gaussian tails. The existence of such anomalous tails has been observed in optical experiments $[4,5]$ and in numerical simulations [6]. From the simulations, one expects a crossover to a lognormal distribution on entering the localized regime.
Since the theory of Refs. [2,3] is based on a perturbation expansion in the small parameter $L / N l$, it cannot describe this crossover which occurs when $L / N l \simeq 1$.

It is the purpose of the present paper to provide a nonperturbative calculation of $P\left(T_{m n}\right)$ and $P\left(T_{n}\right)$, which is valid all the way from the diffusive into the localized regime, and which shows how the Rayleigh and Gaussian distributions of $T_{m n}$ and $T_{n}$ evolve into the same lognormal distribution as $L$ increases beyond the localization length $\xi \simeq N l$. We expect that $P(T)$ also evolves from a Gaussian to a lognormal distribution, but our calculation applies only to the plane-wave transmittances $T_{m n}$ and $T_{n}$, and not to the transmittance $T$ for diffuse illumination. For technical reasons, we need to assume that time-reversal symmetry is broken by some magneto-optical effect. Similar results are expected in the presence of time-reversal symmetry, but then a nonperturbative calculation becomes much more involved. We make essential use of the quasi-one-dimensionality of the waveguide (length $L$ much greater than width $W$ ) and assume weak disorder (mean free path $l$ much greater than wavelength $\lambda$ ). The localization which occurs in unbounded media when $l \leqslant \lambda$ requires a very different nonperturbative approach [7].

A related problem of experimental interest is the transmittance of a cavity coupled to two $N$-mode waveguides without disorder. If the cavity has an irregular shape, it has a complicated "chaotic" spectrum of eigenmodes. At the end of the paper we compute $P\left(T_{m n}\right)$ and $P\left(T_{n}\right)$ for such a chaotic cavity and contrast the results with the disordered waveguide, which we consider first.

Our calculation applies results from random-matrix theory for the statistics of the transmission matrix. This matrix $t=u \sqrt{\tau} v$ is the product of two unitary matrices $u$ and $v$, and a matrix $\tau=\operatorname{diag}\left(\tau_{1}, \tau_{2}, \ldots, \tau_{N}\right)$ containing the transmission eigenvalues. It describes the transmission of electrons or electromagnetic radiation, to the extent that the effects of electron-electron interaction or polarization can be disregarded. The two plane-wave transmittances which we consider are

$$
T_{m n}=\sum_{k, l} u_{m k} u_{m l}^{*} v_{k n} v_{l n}^{*} \sqrt{\tau_{k} \tau_{l}}, \quad T_{n}=\sum_{k}\left|v_{k n}\right|^{2} \tau_{k}
$$

We seek the probability distributions 


$$
\begin{gathered}
P\left(\mathscr{F}_{m n}\right)=\left\langle\delta\left(\mathscr{T}_{m n}-N^{2} T_{m n}\right)\right\rangle, \\
P\left(\mathscr{T}_{n}\right)=\left\langle\delta\left(\mathscr{T}_{n}-N T_{n}\right)\right\rangle,
\end{gathered}
$$

of the normalized transmittances $\mathscr{T}_{m n}=N^{2} T_{m n}$ and $\mathscr{T}_{n}=N T_{n}$. (These conventions differ by a factor $l / L$ with Refs. $[2,3]$.) The brackets \langle\rangle denote an average over the disorder. In the quasi-one-dimensional limit of a waveguide which is much longer than wide, the matrices $u$ and $v$ are uniformly distributed in the unitary group [8]. The joint probability distribution of the transmission eigenvalues evolves with increasing $L$ according to the Dorokhov-MelloPereyra-Kumar (DMPK) equation [9]. The average can be performed in two steps, first over $u$ and $v$, and then over the transmission eigenvalues $\tau_{k}$.

The first step was done by Kogan and Kaveh [3]. The result is an expression for the Laplace transform of $P\left(\mathscr{T}_{n}\right)$,

$$
F(s)=\int_{0}^{\infty} d \mathscr{T}_{n} \exp \left(-s \mathscr{T}_{n}\right) P\left(\mathscr{T}_{n}\right)
$$

which in the thick-waveguide limit $(N \rightarrow \infty, L / l \rightarrow \infty$, fixed $N l / L)$ is exactly given by

$$
F(s)=\left\langle\prod_{k}\left(1+s \tau_{k}\right)^{-1}\right\rangle
$$

The same function $F(s)$ also determines $P\left(\mathscr{T}_{m n}\right)$, which in the same limit is related to $P\left(\mathscr{T}_{n}\right)$ by [3]

$$
P\left(\mathscr{T}_{m n}\right)=\int_{0}^{\infty} d \mathscr{T}_{n} \mathscr{T}_{n}^{1} \exp \left(-\mathscr{T}_{m n} / \mathscr{T}_{n}\right) P\left(\mathscr{T}_{n}\right)
$$

The next step, which is the most difficult one, is to average over the transmission eigenvalues in Eq. (5). The result depends on whether time-reversal symmetry is present or not (indicated by $\beta=1$ or 2 , respectively). In Refs. [2,3], $\ln F$ was evaluated to leading order in $L / N l$, under the assumption that the waveguide length $L$ is much less than the localization length $\xi \simeq N l$. Here we relax this assumption.

We consider the case of broken time-reversal symmetry $(\beta=2)$. Then the probability distribution of the $\tau_{k}$ 's is known exactly, in the form of a determinant of Legendre functions $P_{\nu}$ [10]. Still, to compute expectation values with this distribution is in general a formidable problem. It is a lucky coincidence that the average (5) which we need can be evaluated exactly. This was shown by Rejaei [11], using a field-theoretic approach which leads to a supersymmetric nonlinear $\sigma$ model [12]. It was recently proven [13] that this supersymmetric theory is equivalent to the DMPK equation used in Ref. [10]. From Rejaei's general expressions we find

$$
\begin{gathered}
F(s)=1-2 s \sum_{p=0}^{\infty} \int_{0}^{\infty} d k f_{p}(k) \tanh \left(\frac{1}{2} \pi k\right) P_{(1 / 2)(t k-1)}(1+2 s), \\
f_{p}(k)=\frac{(2 p+1) k}{(2 p+1)^{2}+k^{2}} \exp \left(-\frac{L\left[(2 p+1)^{2}+k^{2}\right]}{4 N l}\right) .
\end{gathered}
$$

Inversion of the Laplace transform (4) yields $P\left(\mathscr{T}_{n}\right)$,
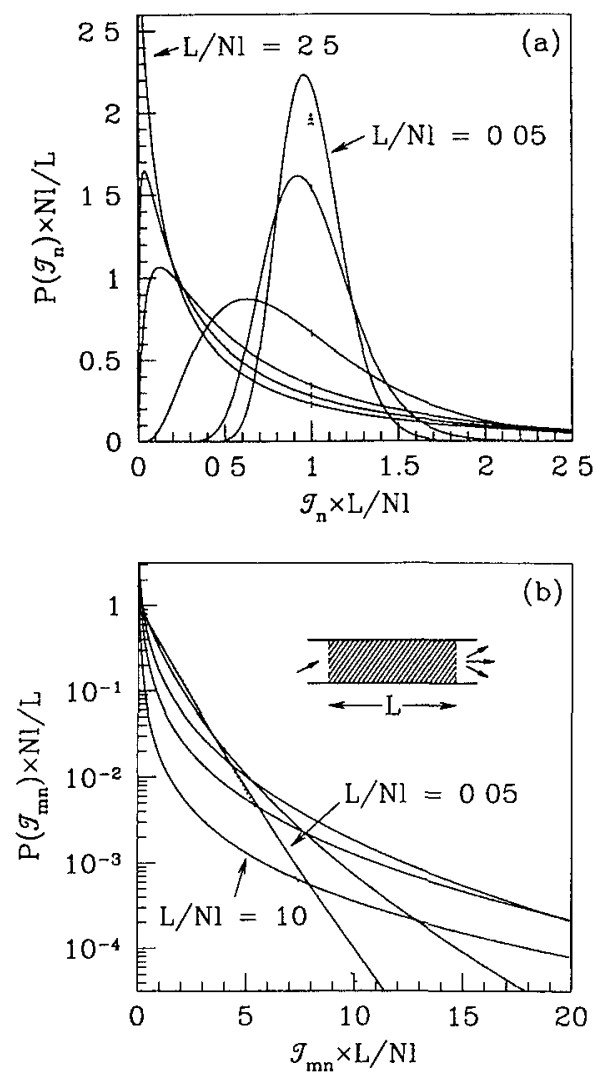

FIG. 1. Distributions of (a) $\mathscr{T}_{n} \equiv N T_{n}$ for $L / N l=0.05,0.1,0.5$, $1.5,2.0$, and 2.5, and (b) $\mathscr{F}_{m n} \equiv N^{2} T_{m n}$ for $L / N l=0.05,0.5,2.5,5$, and 10. Computed from the exact $\beta=2$ expressions (6) and (7). The dotted curves are the limits $L / N l \rightarrow 0$ of an infinitely narrow Gaussian in (a) and an exponential distribution in (b) (note the logarithmic scale). The inset in (b) shows the waveguide geometry considered (disordered region is shaded).

$P\left(\mathscr{T}_{n}\right)=\sum_{p=0}^{\infty} \int_{0}^{\infty} d k f_{p}(k) \sinh \left(\frac{1}{2} \pi k\right) \frac{\partial}{\partial \mathscr{T}_{n}} \frac{2 K_{(1 / 2) l k}\left(\frac{1}{2} \mathscr{T}_{n}\right)}{\left(\pi^{3} \mathscr{F}_{n} e^{\mathscr{T} n}\right)^{1 / 2}}$

where $K_{\nu}$ is the Macdonald function. One further integration gives $P\left(\mathscr{T}_{m n}\right)$, in view of Eq. (6). Results are plotted in Fig. 1. The large $\mathscr{T}_{n}$ and $\mathscr{T}_{m n}$ tails are

$$
\begin{gathered}
P\left(\mathscr{T}_{m n}\right) \propto \mathscr{T}_{m n}^{3 / 4} e^{-2 \sqrt{\mathscr{T}} m n}, \quad \mathscr{T}_{m n} \gg 1,(N l / L)^{2}, \\
P\left(\mathscr{T}_{n}\right) \propto \mathscr{T}_{n}^{1} e^{-\mathscr{T}_{n}}, \quad \mathscr{T}_{n} \gg 1, N l / L .
\end{gathered}
$$

It is worth noting that Fyodorov and Mirlin [14] found the same tail as Eq. (8a) for the distribution of the local density of electronic states in a closed disordered wire. It is not clear to us whether this coincidence is accidental.

The diffusive and localized limits can be computed from Eq. (5) by using the known asymptotic form of the distribution of the $\tau_{k}$ 's. In contrast to the full result (7), which holds for $\beta=2$ only, the following asymptotic expressions hold for any $\beta$. In the diffusive regime, for $L \ll N l$, we may expand $\ln F$ in cumulants of the linear statistic $A=\Sigma_{k} \ln \left(1+s \tau_{k}\right)$ : 


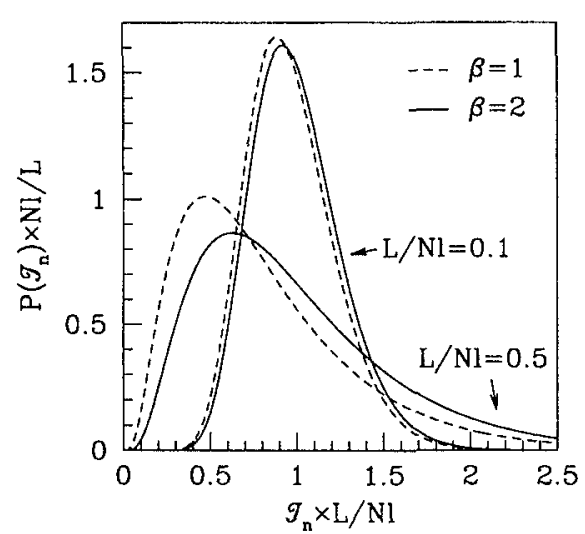

FIG. 2. Distribution of $\mathscr{T}_{n}$ calculated from the perturbation expansion (9), (10), for $\beta=1,2$ and $L / N l=0.1,0.5$.

$$
\ln F(s) \equiv \ln \left\langle e^{-A}\right\rangle=-\langle A\rangle+\frac{1}{2} \operatorname{var} A+O(L / N l)
$$

The mean and variance of $A$ can be computed from the general formulas of Refs. $[10,15,16]$ :

$$
\begin{aligned}
& \langle A\rangle=\frac{N l}{L} \operatorname{arcsinh}^{2} \sqrt{s}+\frac{2-\beta}{4 \beta} \ln \left[\frac{\operatorname{arcsinh}^{2} \sqrt{s}}{s(1+s)}\right], \\
& \operatorname{var} A=-\frac{1}{\beta}\left[\ln (1+s)+6 \ln \left(\frac{\operatorname{arcsinh} \sqrt{s}}{\sqrt{s}}\right)\right],
\end{aligned}
$$

valid up to corrections of order $L / N l$. To leading order in $L / N l$ one has the $\beta$-independent result of Refs. [2,3], yielding Gaussian and Rayleigh statistics for $L / N l \rightarrow 0$. The $\beta$-dependent terms in Eqs. (10) are the first corrections due to localization effects. In Fig. 2 we plot $P\left(\mathscr{T}_{n}\right)$ resulting from Eqs. (9) and (10). The $\beta$-independent result of Refs. $[2,3]$ (not shown) is very close to the $\beta=2$ curve. This figure indicates that the $\beta$ dependence is essentially quantitative rather than qualitative.

In the opposite, localized regime $(L \gg N l)$, only a single transmission eigenvalue contributes significantly to Eq. (5). This largest eigenvalue $\tau$ has the lognormal distribution [17]

$$
P(\ln \tau)=\left(\frac{\beta N l}{8 \pi L}\right)^{1 / 2} \exp \left[-\frac{\beta N l}{8 L}\left(\frac{2 L}{\beta N l}+\ln \tau\right)^{2}\right] .
$$

It follows that $\ln \mathscr{T}_{m n}$ and $\ln \mathscr{T}_{n}$ are also distributed according to Eq. (11) in the localized regime. The approach to a common lognormal distribution as $L / N l$ increases is illustrated in Fig. 3, using the exact $\beta=2$ result of Eq. (7).

We contrast these results for a disordered waveguide with those for a chaotic cavity, attached to two $N$-mode leads without disorder. Following Ref. [18] we assume that the $2 N \times 2 N$ scattering matrix of the cavity is distributed uniformly in the unitary group if $\beta=2$ or in the subset of unitary and symmetric matrices if $\beta=1$. Then $P\left(T_{m n}\right)$ and $P\left(T_{n}\right)$ follow from general formulas [19] for the distribution of matrix elements in these so-called "circular" ensembles. For $\beta=2$ the result is

$$
P\left(T_{m n}\right)=(2 N-1)\left(1-T_{m n}\right)^{2 N-2},
$$

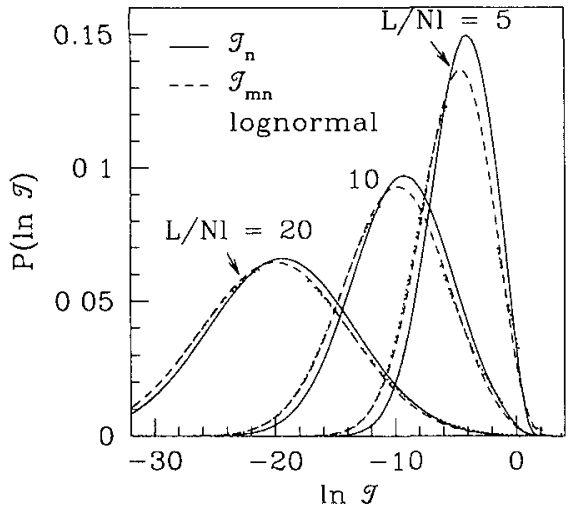

FIG. 3. Distributions of $\mathscr{T}_{n}$ and $\mathscr{T}_{m n}$ for $\beta=2$ and $L / N l=5,10,20$, computed from Eqs. (6) and (7). The dotted curve is the lognormal distribution (11) which is approached as $L / N l \rightarrow \infty$.

$$
P\left(T_{n}\right)=\frac{1}{2} N\left(\begin{array}{c}
2 N \\
N
\end{array}\right)\left[T_{n}\left(1-T_{n}\right)\right]^{N-1}
$$

For $\beta=1$ Eq. (12a) should be multiplied by $\frac{1}{2} F\left(N-\frac{1}{2}, 1 ; 2 N-1 ; 1-T_{m n}\right)$ and Eq. (12b) by $\frac{1}{2} F\left(N-\frac{1}{2}, 1\right.$; $\left.N ; 1-T_{n}\right)$, where $F$ is the hypergeometric function. These are exact results for any $N$. If $N \rightarrow \infty, P\left(T_{m n}\right)$ is an exponential distribution with mean $1 / 2 N$, and $P\left(T_{n}\right)$ is a Gaussian with mean $1 / 2$ and variance $1 / 8 N$. This is similar to the disordered waveguide, with $N$ playing the role of $N l / L$. As shown in Fig. 4, the distributions for $N$ of order unity are quite different from those in a disordered waveguide with $N l / L$ of order unity. For $N=1$ the distinction between $T_{m n}, T_{n}$, and $T$ disappears and we recover the results of Ref. [18].

In conclusion, we have presented a nonperturbative calculation of the distributions of the plane-wave transmittances $T_{m n}$ and $T_{n}$ through a disordered waveguide without timereversal symmetry, which shows how the distributions cross over from Rayleigh and Gaussian statistics in the diffusive regime, to a common lognormal distribution in the localized regime. Qualitatively different distributions are obtained if the disordered region is replaced by a chaotic cavity. Existing experiments have been mainly in the regime $L \ll N l$ where the perturbation theory of Refs. [2,3] applies. If the absorp-

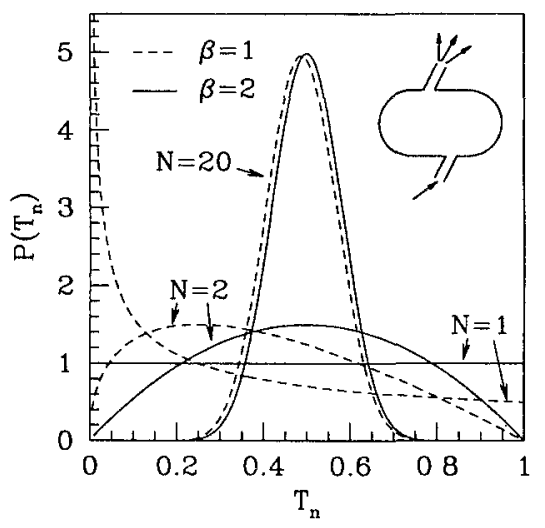

FIG. 4. Distribution of $T_{n}$ for a chaotic cavity attached to two $N$-mode leads (inset). The curves are computed from Eq. (12b), for $\beta=1,2$ and $N=1,2,20$. 
tion of light in the waveguide can be reduced sufficiently, it should be possible to enter the regime $L \simeq N l$ where perturbation theory breaks down and the crossover to lognormal statistics is expected.
This research was supported by the "Nederlandse Organisatie voor Wetenschappelijk Onderzoek" (NWO) and by the "Stichting voor Fundamenteel Onderzoek der Materie" (FOM).
[1] M. E. Gertsenshtein and V. B. Vasil'ev, Teor. Veroyatn. Primen. 4, 424 (1959); 5, 3(E) (1960) [Theor. Probab. Appl. 4, 391 (1959); 5, 340(E) (1960)].

[2] Th. M. Nieuwenhuizen and M. C. W. van Rossum, Phys. Rev. Lett. 74, 2674 (1995).

[3] E. Kogan and M. Kaveh, Phys. Rev. B 52, 3813 (1995).

[4] A. Z. Genack and N. Garcia, Europhys. Lett. 21, 753 (1993).

[5] J. F. de Boer, M. C. W. van Rossum, M. P. van Albada, Th. M. Nieuwenhuizen, and A. Lagendijk, Phys. Rev. Lett. 73, 2567 (1994).

[6] I. Edrei, M. Kaveh, and B. Shapiro, Phys. Rev. Lett. 62, 2120 (1989).

[7] B. L. Al'tshuler, V. E. Kravtsov, and I. V. Lerner, in Mesoscopic Phenomena in Solids, edited by B. L. Al'tshuler, P. A. Lee, and R. A. Webb (North-Holland, Amsterdam, 1991); B. A. Muzykantskiĭ and D. E. Khmel'nitskiı̌, Phys. Rev. B 51, 5480 (1995); V. I. Fal'ko and K. B. Efetov, Report Nos. (cond-mat/ 9503096, 9507091); A. D. Mirlin, Pis'ma Zh. Eksp. Teor. Fiz. 62, 583 (1995) [JETP Lett. 62, 603 (1995)]; Phys. Rev. B 53, 1186 (1996).

[8] P. A. Mello, E. Akkermans, and B. Shapiro, Phys. Rev. Lett. 61, 459 (1988).
[9] O. N. Dorokhov, Pis'ma Zh. Éksp. Teor. Fiz. 36, 259 (1982) [JETP Lett. 36, 318 (1982)]; P. A. Mello, P. Pereyra, and N. Kumar, Ann. Phys. (N.Y.) 181, 290 (1988).

[10] C. W. J. Beenakker and B. Rejaei, Phys. Rev. Lett. 71, 3689 (1993); Phys. Rev. B 49, 7499 (1994).

[11] B. Rejaei, Phys. Rev. B (to be published).

[12] K. B. Efetov, Adv. Phys. 32, 53 (1983).

[13] P. W. Brouwer and K. Frahm, Phys. Rev, B 53, 1490 (1996).

[14] Y. V. Fyodorov and A. D. Mirlin, Int. J. Mod. Phys. B 27, 3795 (1994).

[15] C. W. J. Beenakker, Phys. Rev. B 49, 2205 (1994).

[16] J. T. Chalker and A. M. S. Macêdo, Phys. Rev. Lett. 71, 3693 (1993); Phys. Rev. B 49, 4695 (1994).

[17] A. D. Stone, P. A. Mello, K. A. Muttalib, and J.-L. Pichard, in Mesoscopic Phenomena in Sollds, edited by B. L. Al'tshuler, P. A. Lee, and R. A. Webb (North-Holland, Amsterdam, 1991).

[18] H. U. Baranger and P. A. Mello, Phys. Rev. Lett. 73, 142 (1994); R. A. Jalabert, J.-L. Pichard, and C. W. J. Beenakker, Europhys. Lett. 27, 255 (1994).

[19] P. Pereyra and P. A. Mello, J. Phys. A 16, 237 (1983). 